\title{
Intersectin links WNK kinases to endocytosis of ROMK1
}

\author{
Guocheng He, Hao-Ran Wang, Shao-Kuei Huang, and Chou-Long Huang \\ Department of Medicine, University of Texas Southwestern Medical Center, Dallas, Texas, USA.
}

\begin{abstract}
With-no-lysine (WNK) kinases are a novel family of protein kinases characterized by an atypical placement of the catalytic lysine. Mutations of 2 family members, WNK1 and WNK4, cause pseudohypoaldosteronism type 2 (PHA2), an autosomal-dominant disease characterized by hypertension and hyperkalemia. WNK1 and WNK4 stimulate clathrin-dependent endocytosis of renal outer medullar potassium 1 (ROMK1), and PHA2causing mutations of WNK4 increase the endocytosis. How WNKs stimulate endocytosis of ROMK1 and how mutations of WNK4 increase the endocytosis are unknown. Intersectin (ITSN) is a multimodular endocytic scaffold protein. Here we show that WNK1 and WNK4 interacted with ITSN and that the interactions were crucial for stimulation of endocytosis of ROMK1 by WNKs. The stimulation of endocytosis of ROMK1 by WNK1 and WNK4 required specific proline-rich motifs of WNKs, but did not require their kinase activity. WNK4 interacted with ROMK1 as well as with ITSN. Disease-causing WNK4 mutations enhanced interactions of WNK4 with ITSN and ROMK1, leading to increased endocytosis of ROMK1. These results provide a molecular mechanism for stimulation of endocytosis of ROMK1 by WNK kinases.
\end{abstract}

\section{Introduction}

With-no-lysine (WNK) kinases are a novel family of large serinethreonine protein kinases named for an atypical placement of the catalytic lysine (1). Four family members, WNK1-WNK4, have been identified (1-3). WNK1 spans over 2,100 amino acids and contains an approximately 270 -amino acid kinase domain located near the amino terminus (1). WNK2, WNK3, and WNK4 are products of different genes and are 1,200-1,600 amino acids in length (1-3). The 4 WNK kinases share a conserved kinase domain with 85\%-90\% sequence identity, an autoinhibitory domain, 1-2 coiledcoil domains, and multiple PXXP proline-rich motifs for potential protein-protein interaction (1-3). Little sequence identity exists beyond these conserved domains and motifs.

Pseudohypoaldosteronism type 2 (PHA2), also known as Gordon's syndrome or familial hyperkalemic hypertension, is an autosomal-dominant disease characterized by hypertension and hyperkalemia (4). Positional cloning has recently identified mutations of WNK1 and WNK4 as causes for PHA2 (3). Mutations of the WNK1 gene in PHA2 are large deletions within the first intron that cause its increased expression. PHA2-causing mutations in the WNK4 gene are missense mutations in the coding sequence outside the protein kinase domain.

WNK1 is widely expressed in multiple spliced forms $(1,5,6)$. The full-length WNK1, consisting of over 2,100 amino acids (referred to herein as long WNK1), is ubiquitous (1). A shorter WNK1 transcript encoding a polypeptide lacking the aminoterminal 1-437 amino acids (including most of the kinase domain) of long WNK1 is expressed exclusively in the kidney (referred to herein as kidney-

Nonstandard abbreviations used: AQP2, aquaporin-2; CCD, cortical collecting duct; CCV, clathrin-coated vesicle; CNT, connecting tubule; DCT, distal convoluted tubule; EH, Eps15 homology; GST, glutathione S-transferase; H6, hexahistidine; IRK1, inward-rectifying $\mathrm{K}^{+}$1; ITSN, intersectin; ITSN1s, ITSN1 short form; NCX1, sodium-calcium exchanger isoform 1; OSR1, oxidative stress-response kinase 1; PHA2, pseudohypoaldosteronism type 2; ROMK1, renal outer medullar potassium 1; SH3, Src-homology 3; WNK, with-no-lysine.

Conflict of interest: The authors have declared that no conflict of interest exists. Citation for this article: J. Clin. Invest. 117:1078-1087 (2007). doi:10.1172/JCI30087. specific WNK1) $(5,6)$. Mutations in the WNK1 gene in PHA2 cause increased expression of long WNK1 (3). Whether expression of kidney-specific WNK1 is altered in PHA2 is currently unknown.

The discovery of WNK1 and WNK4 gene mutations as causes of PHA2 has led to recent advances in the understanding of the pathogenesis of hypertension and hyperkalemia of PHA2. It was reported that in Xenopus oocytes, WNK4 inhibits the activity of the sodium chloride cotransporter and that PHA2-causing WNK4 mutants fail to inhibit the sodium chloride cotransporter $(7,8)$. Others reported that in cultured epithelial cells, WNK4 phosphorylates tight-junction proteins claudins 1-4 and regulates the paracellular chloride permeability $(9,10)$. The paracellular chloride permeability is greater in cells expressing WNK4 mutants than in cells expressing wild-type proteins $(9,10)$. These results suggest that hypertension in patients with WNK4 mutations is caused by increased $\mathrm{NaCl}$ reabsorption through the $\mathrm{Na}-\mathrm{Cl}$ cotransporter and the paracellular pathway. It has also been reported that expression of long WNK1 abolishes the inhibition of the sodium chloride cotransporter caused by WNK4 (7). Long WNK1 activates serum- and glucocorticoid-inducible kinase, leading to activation of the epithelial $\mathrm{Na}^{+}$channel (ENaC; ref. 11). Thus, hypertension in PHA2 patients with WNK1 mutations may be caused by increased activity of $\mathrm{Na}-\mathrm{Cl}$ cotransporter and $\mathrm{ENaC}$.

Wild-type WNK4 inhibits the renal outer medullar potassium 1 (ROMK1) channel, and PHA2-causing WNK4 mutants exhibit increased inhibition of ROMK1 (12). Several recent studies have reported that long WNK1 inhibits ROMK1 (13-15). Interestingly, kidney-specific WNK1 by itself does not inhibit ROMK1, but antagonizes the inhibition of ROMK1 caused by long WNK1 (13, 14). These results suggest that inhibition of $\mathrm{K}^{+}$secretion through ROMK1 may contribute to hyperkalemia caused by mutations of WNK1 and WNK4 in PHA2. ROMK1 channels undergo clathrinmediated endocytosis, which plays an important role in the regulation of $\mathrm{K}^{+}$secretion by dietary $\mathrm{K}^{+}$intake (16-18). The inhibition of ROMK1 by long WNK1 and WNK4 occurs, at least in part, by increasing endocytosis of the channel (12-15). How long WNK1 and WNK4 mediate endocytosis of ROMK1 and how mutations of WNKs increase the endocytosis are unknown. 
A

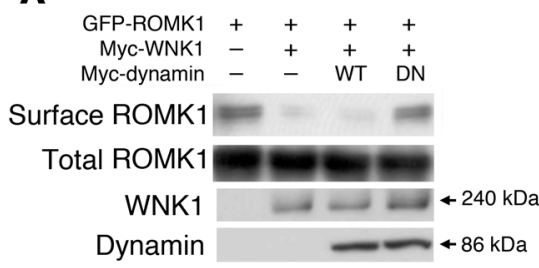

B

WB: Anti-WNK1

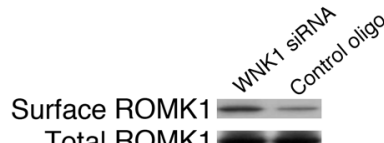

Total ROMK1

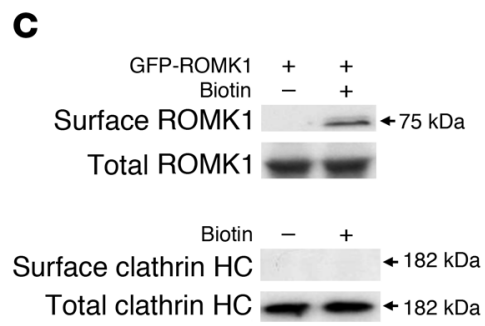

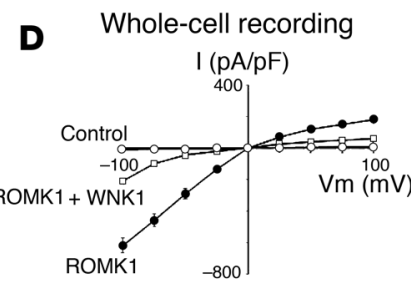

Cell-attached single channel

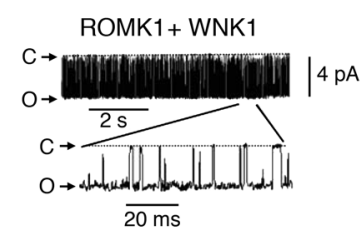

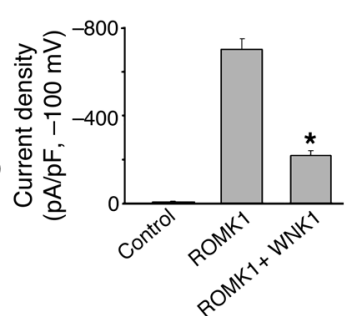

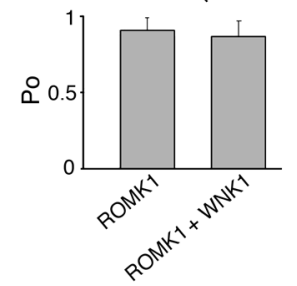

Figure 1

Effects of long WNK1 on ROMK1. (A) Long WNK1 decreases surface abundance of GFP-ROMK1 via a dynamin-dependent mechanism. Surface abundance was measured by biotinylation assays. Equal expression of the total ROMK1 in transfected cells was confirmed by Western blot analysis. Expression of Myc-tagged full-length long WNK1 and dynamin-2 (WT or dominant-negative; DN) was examined by an anti-myc antibody. The molecular size of proteins is shown. (B) Knockdown of endogenous long WNK1 by siRNA (top) increased surface abundance of ROMK1 (bottom). HEK cells were transfected with GFP-ROMK1 alone. Control oligo, control oligonucleotides. (C) Validation of the integrity of biotinylation assay. Cells were transfected with GFP-ROMK1 and incubated with or without biotin before lysis. Biotinylated proteins were precipitated by streptavidin beads. HC, heavy chain. (D) Effects of long WNK1 on whole-cell and single ROMK1 channel activity. Top left: Current-voltage relationship of whole-cell ROMK1 currents. Top right: Long WNK1 decreased whole-cell ROMK1 currents. Bottom: Long WNK1 had no effects on single-channel conductance (at $-100 \mathrm{mV}$ ) or open probability (Po) of ROMK1. Open probability was measured based on approximately 15 minutes recording from patches containing 1 active channel. ${ }^{*} P<0.05$ vs ROMK alone. C, closed state; O, open state.

Endocytosis via clathrin-coated vesicles (CCVs) involves concerted actions of many proteins $(19,20)$. Among these, endophilin, amphiphysin, and intersectin (ITSN) contain Src-homology 3 (SH3) domains that can interact with PXXP proline-rich motifs (21). In the present study, we found that long WNK1 and WNK4 specifically interacted with the endocytic scaffold protein ITSN. The interaction required specific proline-rich motifs of WNKs, but not their kinase activity. Knockdown of ITSN prevented the stimulation of endocytosis of ROMK1 by long WNK1 and WNK4. WNK4 interacted with ROMK1 as well as with ITSN. Diseasecausing mutations enhance interactions of WNK4 with ITSN and ROMK1, leading to increased endocytosis of ROMK1. These results provide a molecular mechanism for stimulation of endocytosis of ROMK1 by WNK kinases.

\section{Results}

Long WNK1 decreases surface abundance of ROMK1 via a dynamin- and clathrin-dependent mechanism. The abundance of ROMK1 in the plasma membrane is regulated by constitutive clathrin-mediated endohas multiple PXXP type proline-rich motifs $(1,2)$ that can interact with SH3 domains (21). We hypothesized that long WNK1 stimulates clathrin-mediated endocytosis of ROMK1 by interacting with SH3-containing endocytic accessory proteins. We examined interactions between long WNK1 and SH3 domains of ITSN, endophilin, and amphiphysin. In humans, rats and mice, there are 2 ITSN genes, ITSN1 and ITSN2 (22-24). Each ITSN gene gives rise to alternatively spliced long and short forms. The short form of ITSN1 (ITSN1s) is ubiquitous $(23,24)$ and contains 2 Eps15 homology (EH) domains, a central coiled-coil region, and 5 consecutive SH3 domains (SH3A-SH3E; Figure 2A). The long form additionally contains Dbl homology, pleckstrin homology, and C2 domains in the C terminus (22-24). Amino acids 1-491 of long WNK1 are sufficient for regulation of ROMK1 (13). We used purified bacterial glutathione $S$-transferase (GST) fusion proteins containing ITSN1 SH3A-SH3E and the SH3 domains of amphiphysin and endophilin to pull down myc-tagged WNK1 $1_{1-491}$ from lysates of transfected HEK cells. We found that ITSN1 SH3C pulled down WNK1 $1_{1-491}$ (Figure 2B). The interaction between 
A
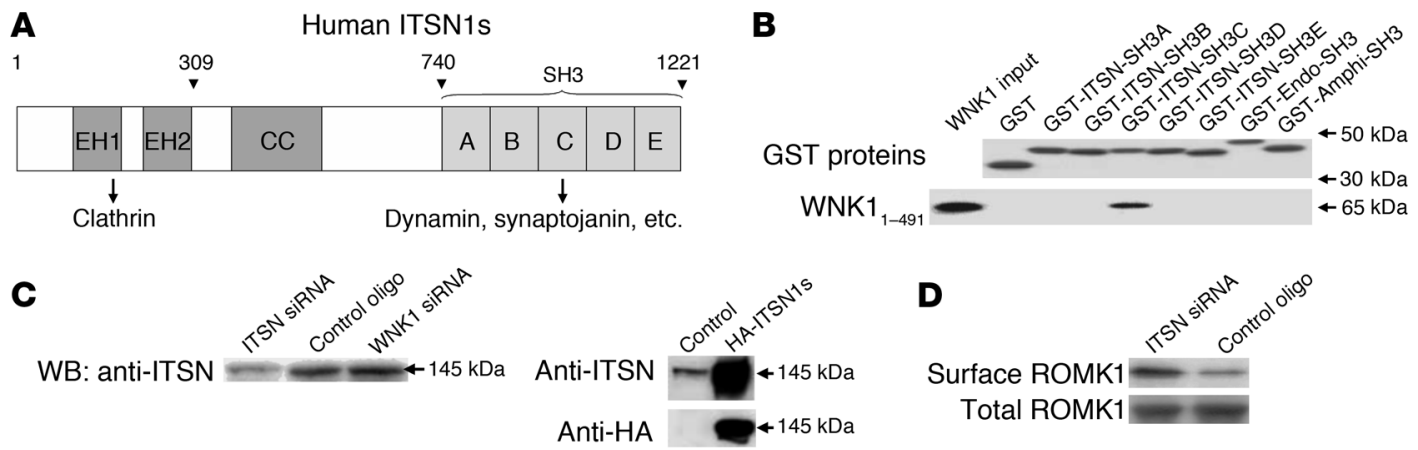

$\mathbf{E}$

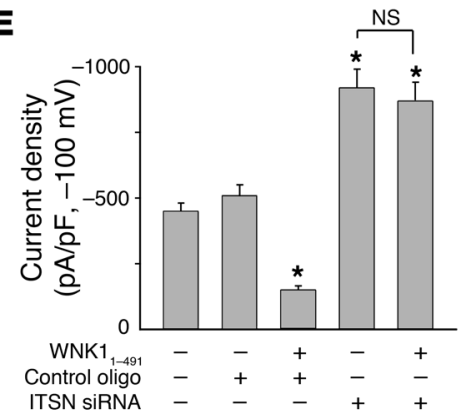

$\mathbf{F}$

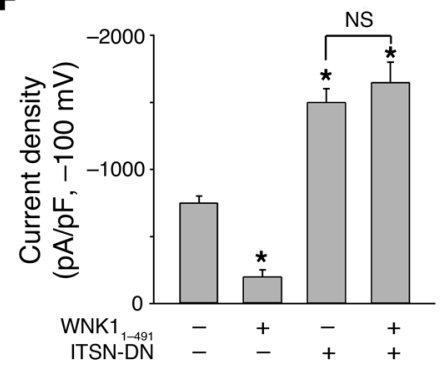

Figure 2

ITSN is important for long WNK1 regulation of ROMK1. (A) Domain structure of ITSN1s. It contains 2 EH domains, a central coiled-coil (CC) region, and 5 consecutive SH3 domains. The EH domains anchor ITSN to clathrin coat. The SH3 domains recruit dynamin, synaptojanin, and other endocytic proteins. (B) Amino acids 1-491 of long WNK1 interacted specifically with the ITSN SH3 C domain. GST fusion proteins were used to pull down myc-tagged WNK1 $1_{1-491}$ from transfected cell lysates. Western blot analysis was performed with anti-GST and anti-myc antibodies, respectively. Amphi, amphiphysin; Endo, endophilin. (C) Evidence for knockdown of endogenous ITSN1. Endogenous ITSN1s was detected by an anti-ITSN antibody. Western blot analysis of lysates from mock-transfected (control) cells and cells transfected with HA-tagged ITSN1s probed by anti-ITSN and anti-HA antibodies is shown on the right. (D) Knockdown of ITSN1 increased surface abundance of ROMK1. (E) Knockdown of ITSN1 increased baseline whole-cell ROMK1 current density and prevented the decrease caused by WNK $1_{1-491}$. Cells were transfected with ROMK1 plus indicated constructs. (F) Overexpression of dominant-negative ITSN1 increased baseline whole-cell ROMK1 current density and prevented the decrease caused by WNK1 $1_{1-491 .}{ }^{*} P<0.05$ versus ROMK1 alone.

ITSN1 SH3C and WNK1 1-491 was highly specific, as other SH3 domains of ITSN1 and the SH3 domain of amphiphysin and endophilin failed to pull down WNK1 $1_{1-491}$.

The importance of interaction with ITSN in regulating the surface abundance of ROMK1 by long WNK1 was further examined by using siRNA to knock down endogenous ITSN. Knockdown of endogenous ITSN1s in HEK cells by siRNA was evident from Western blot analysis using an anti-ITSN antibody (Figure 2C). This antibody recognized endogenous ITSN1s and transfected HA-tagged ITSN1s (Figure 2C). The transfected HA-tagged ITSN1s was identified by an anti-HA antibody. Cotransfection with siRNA for ITSN1 (but not control oligonucleotides) increased the surface abundance of ROMK1 (Figure 2D). Furthermore, knockdown of ITSN1 by siRNA increased basal ROMK1 current (i.e., in the absence of exogenous long WNK1) and prevented the inhibition of ROMK1 by exogenous long WNK1 (Figure 2E). Similarly, overexpression of ITSN1 SH3A-SH3E $(25,26)$ produced a dominant-negative effect to prevent long WNK1 inhibition of ROMK1 (Figure 2F).

Proline-rich motifs in the N terminus of long WNK1 are critical for interaction with ITSN and for regulation of ROMK1. Rat long WNK1 is 2,126 amino acids long (Figure $3 \mathrm{~A}$ and ref. 1). It contains a kinase domain located near the amino terminus (e.g., amino acids 218-491 of rat long WNK1). To further identify determinants of long WNK1 in the regulation of ROMK1, we compared the effects of amino acids 1-119, amino acids 1-491, amino acids 218-491, and full-length long WNK1. We found that WNK1 $1_{1-119}$ as well as $\mathrm{WNK} 1_{1-491}$ and full-length long WNK1 inhibited ROMK1 (Figure 3B). The construct containing the kinase domain of WNK1, WNK1 $1_{218-491}$, did not cause inhibition of ROMK1 (Figure 3B). Expression and kinase activity of WNK1 $1_{218-491}$ were demonstrated (data not shown). Amino acids 1-119 of long WNK1 contain 3 PXXP proline-rich motifs at P94, P103, and P114 (Figure 3A). We examined the role of these PXXP motifs in the regulation of ROMK1. Compared with wildtype WNK1 $1_{1-491}$, single mutants of P94A, P103A, and P114A each caused partial inhibition of ROMK1 currents (Figure 3C). The triple PXXP mutant of WNK1 $1_{1-491}$ failed to inhibit ROMK1 (Figure 3C). Biochemical interactions between ITSN1 SH3C and various long WNK1 constructs were examined in pulldown assays using GSTITSN-SH3C. We found that WNK $1_{1-119}$ interacted with ITSN SH3C and that the triple PXXP mutant failed to interact with ITSN SH3C (Figure 3D). These results support the idea that interactions between the $\mathrm{N}$-terminal proline-rich motifs and ITSN are crucial for long WNK1 regulation of ROMK1. As kidney-specific WNK1 lacks amino acids $1-119$ of long WNK1 $(5,6)$, our current results also support previous reports that kidney-specific WNK1 by itself has no effects on ROMK1 channels $(13,14)$.

WNK1 kinase activity is not required for regulation of ROMK1. The above results indicate that amino acids 1-119 of long WNK1 are sufficient for regulating ROMK1 via ITSN and that kinase activity is not required, yet mutation of the catalytic K233 or a conserved 
A

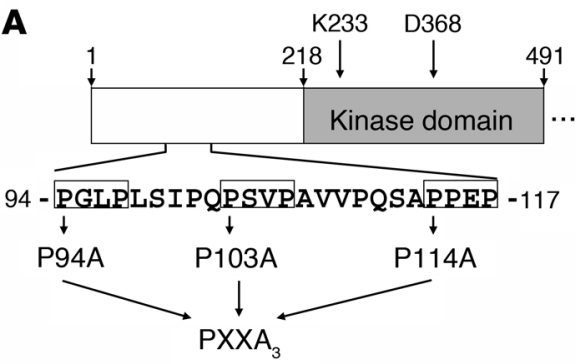

C

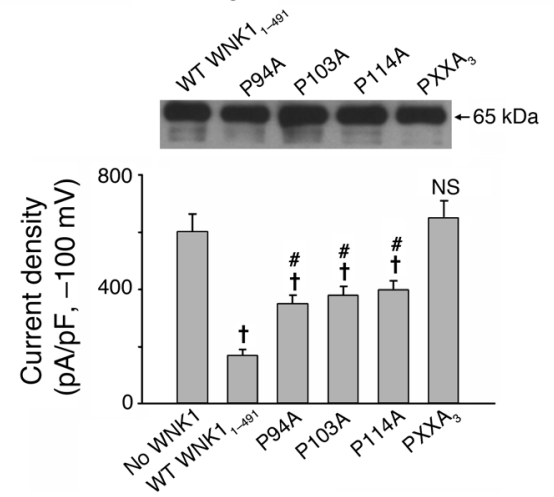

Figure 3

Specific proline-rich motifs of long WNK1 are critical for regulation of ROMK1 via ITSN. (A) The region of amino acids $1-119$ of rat long WNK1 contains 3 PXXP motifs conserved among mouse,

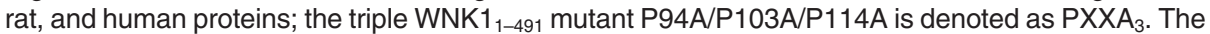
catalytic K233 and conserved D368 of the WNK1 kinase domain are shown. (B) Effects of various $\mathrm{N}$-terminal long WNK1 fragments on ROMK1 current density. Cells were transfected with ROMK1 plus indicated constructs. FL, full-length. ${ }^{*} P<0.05$ versus ROMK1 alone. (C) Effects of various long WNK1 constructs on ROMK1 current density. The concentration that causes a maximal inhibition of ROMK1 current ( $3 \mu \mathrm{g}$ DNA per transfection per 6-well dish) for wild-type WNK1 $1_{1-491}$ and each mutant were used. Equal levels of protein expression for each construct were confirmed by Western blot analysis using an anti-myc antibody. Modified statistics adjusted for multiple comparisons was used (see Methods). ${ }^{\dagger} P<0.01$ versus ROMK1 alone. ${ }^{\#} P<0.01$ versus ROMK1 plus WT WNK1 $1_{1-491}$. NS, not significantly different versus ROMK1 alone. (D) Pulldown of various long WNK1 constructs by GST fusion protein of ITSN SH3C. Cells were transfected with myc-tagged WNK1 $1_{1-119}$, WNK1 $1_{1-491}$, or the triple WNK1 $1_{1-491}$ mutant P94A/P103A/P114A. GST-ITSN-SH3C was used to pull down lysates from transfected cells. Shown are Western blots probed by anti-GST and anti-myc antibodies, respectively. Equal expression of long WNK1 constructs (WNK1 input) was confirmed.

D368 in the WNK1 kinase domain abolishes its ability to inhibit ROMK1 $(13,14)$. In addition, we have previously shown that a construct containing amino acids 1-220 of long WNK1 does not inhibit ROMK1 (13). Together, these results suggest that amino acids 120-220 interfere with the ability of amino acids 1-119 of long WNK1 to regulate ROMK1 and that the kinase domain prevents the interference caused by amino acids 120-220. Indeed, we found that coexpression with WNK1 $1_{120-220}$ reversed the effect of WNK1 $1_{1-119}$ on ROMK1 (Figure 4A). By itself, WNK1 $1_{120-220}$ had no effect on ROMK1.

The crystal structure of the kinase domain of WNK1 reveals that K233 and D368 are in close proximity (27). Thus, we hypothesized that K233 and D368 form an ion pair and that the ionic interaction is critical for the kinase domain to prevent the interference of the WNK1-ITSN interaction caused by amino acids 120-220 of long WNK1. We tested this hypothesis by making charge reversal mutations of K233 and D368. Consistent with the notion that K233 and D368 are critical for the catalytic activity of long WNK1, WNK1 $1_{1-491}$ carrying single mutations K233D and D368K and the double mutation K233D/D368K showed no kinase activity (Fig- ure 4B). However, the double mutant decreased ROMK1 currents (Figure 4C) and interacted with ITSN SH3C (Figure 4D). The effect of the double mutant K233D/D368K on ROMK1 was prevented by cotransfection with a dominant-negative dynamin or with siRNA for clathrin heavy chain (data not shown). As positive controls, we found that neither K233D nor D368K regulated ROMK1 (Figure 4C) or interacted with ITSN (Figure 4D). These results provide compelling evidence that long WNK1 regulates ROMK1 endocytosis via ITSN independent of kinase activity.

Interaction with ITSN is crucial for WNK4 regulation of ROMK1. WNK4 spans 1,223 amino acids (Figure 5A and refs. 2, 3). The kinase domains of WNK1 and WNK4 are conserved (1-3). Other conserved domains include an autoinhibitory domain, 2 coiled-coil domains, multiple PXXP proline-rich motifs, and the highly acidic region, where disease-causing mutations of WNK4 are clustered (Figure 5A). To identify region(s) of WNK4 involved in the regulation of ROMK1, we examined the effects of several $\mathrm{N}$-terminal WNK4 fragments. We found that amino acids $1-584$, but not amino acids $1-535$ or amino acids $1-444$, of WNK4 decreased ROMK1 currents (Figure 5A). The region of amino acids 473-584 decreased ROMK1 currents (Figure 5A). The inhibition of ROMK1 current density by WNK $4_{1-584}$ correlated with decreased surface abundance of the channel (Figure 5B). Experiments using GST pulldown assays revealed that ITSN1 SH3A, SH3B, and SH3C interacted with $\mathrm{WNK}_{1-584}$, whereas even at much higher concentrations, ITSN SH3D and SH3E and the SH3 domains of amphiphysin and endophilin, had no interaction with WNK4 $4_{1-584}$ (Figure 5C). In contrast to $\mathrm{WNK}_{1-584}, \mathrm{WNK}_{1-444}$ did not interact with ITSN (Figure 5D). It has previously been reported that WNK4 interacts with the C terminus of ROMK1 (12). As a positive control for the pulldown assay, we found that $\mathrm{WNK}_{1-444}$ interacted with a GST fusion protein containing the C terminus of ROMK1 (Figure 5D). The functional importance of WNK4-ITSN interaction in the regulation of ROMK1 was demonstrated by the finding that overexpression of ITSN1 SH3A-SH3E prevented WNK4 inhibition of ROMK1 (Figure 5E). Similarly, cotransfection with siRNA for ITSN1 prevented WNK4 inhibition of ROMK1 (Figure 5F).

Disease-causing mutations increase WNK4 interaction with ITSN. The above results indicate that amino acids 473-584 of WNK4 are important for interaction with ITSN and inhibition of ROMK1. This region contains 3 conserved PXXP motifs (which begin at P545, P552, and P555) adjacent to disease-causing mutations E559K, D561A, and Q562E (Figure 6A). Disease-causing mutations 

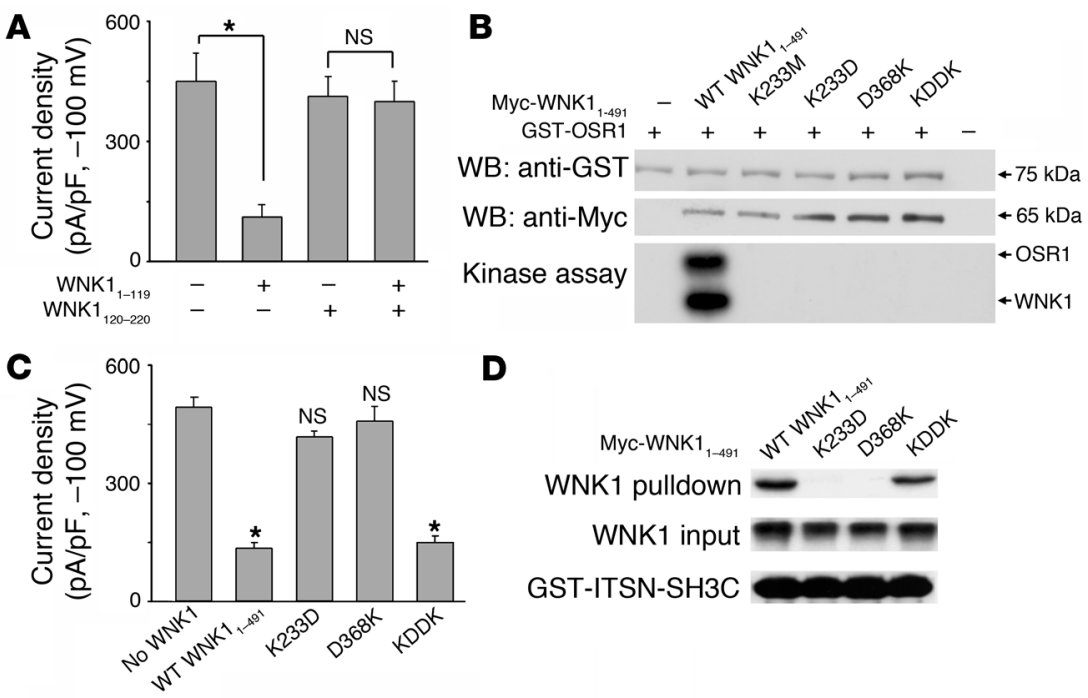

\section{Figure 4}

Role of WNK1 kinase domain in regulation of ROMK1 and interaction with ITSN. (A) Role of WNK1 $1_{120-220}$ in WNK1 $1_{1-119}$ inhibition of ROMK1. ${ }^{*} P<0.05$. (B-D) Effects of K233 and/or D368 mutations of WNK1 ${ }_{1-491}$ on WNK1 kinase activity (B), on ROMK1 current density (C), and on the interaction with ITSN SH3C (D). WNK1 $1_{1-491}$ carrying K233D and D368K single mutations are denoted K233D and D368, respectively. Double WNK1 $1_{1-491}$ mutant K233D/ D368K is denoted KDDK. (B) Wild-type and mutant WNK1 $1_{1-491}$ were immunoprecipitated from lysates of transfected cells and used to phosphorylate purified kinase-dead GSTOSR1. Kinase-dead OSR1 was used here to prevent its autophosphorylation $(37,38)$. Autophosphorylation of WNK1 $1_{1-491}$ is denoted as WNK1. (C) Cells were transfected with ROMK1 plus wild-type WNK $1_{1-491}$ or one of the mutants. ${ }^{*} P<0.05$ versus ROMK1 alone. NS, not significantly different versus ROMK1 alone. (D) GST-ITSN-SH3C was used to pull down wild-type WNK1 $1_{1-491}$ or mutants as described in Figure 3D.

increase the inhibition of ROMK1 by WNK4 (12). We hypothesized that PXXP motifs within this region interact with $\mathrm{SH} 3$ domains of ITSN and that disease-causing mutations enhance the interaction to increase endocytosis of ROMK1. To test this hypothesis, we first examined the role of PXXP motifs within this region in the regulation of ROMK1. Triple mutations of PXXP motifs (Figure 6A) abolished the decrease of surface abundance of ROMK1 mediated by WNK4 $4_{1-584}$ (Figure 6B). In addition, the triple PXXP mutant failed to interact with ITSN SH3A, SH3B, or SH3C (Figure 6C).

We next examined the effects of disease-causing mutations on the surface abundance of ROMK1. We found that disease-causing WNK4 mutants E559K, D561A, and Q562E each decreased the surface abundance of ROMK1 more than wild-type WNK4 $4_{1-584}$ (Figure 6D). The effects of disease-causing mutations on the surface abundance correlated with the effects on ROMK1 current: E559K decreased ROMK1 current more than did wild-type WNK41-584 (Figure 6E). Similarly, the D561A and Q562E mutants decreased ROMK1 current more than did wild-type WNK4 $4_{1-584}$ (data not shown). Triple mutations of PXXP motifs abolished the inhibition of ROMK1 by WNK $4_{1-584}$ and the enhanced inhibition caused by E559K (Figure 6E). Furthermore, disease-causing mutant E599K pulled down more of the SH3 domain of ITSN than did wild-type WNK4 (Figure 6F), as did D561A and Q562E (data not shown).

WNK4 interacts with ROMK1, and disease-causing mutations increase this interaction. WNK4 interacts with the C terminus of ROMK1 (12). We examined potential roles of the interaction in the regulation of ROMK1. The inward-rectifying $\mathrm{K}^{+} 1$ (IRK1) channel has approxi- mately $40 \%$ amino acid sequence identity to ROMK1 (28). In contrast to ROMK1, IRK1 is not present in kidney and is not known to be regulated by clathrin-mediated endocytosis. WNK4 $4_{1-584}$ interacted with the hexahistidine-tagged (H6tagged) C terminus of ROMK1 but not of IRK1 (Figure 7A). WNK4 $4_{1-584}$ had no effect on IRK1 currents (Figure 7A). However, a shorter WNK4 construct, $\mathrm{WNK}_{1-444}$, interacted with the $\mathrm{C}$ terminus of ROMK1 (Figure 5D) despite the lack of ROMK1 inhibition (Figure 5A). Thus, interaction with ROMK1 by itself is not sufficient for WNK4 to inhibit the channel.

We next asked whether interaction with ROMK1 is essential for WNK4 to inhibit the channel. We took advantage of the possibility that WNK4 ${ }_{1-444}$ may be used as a dominantnegative inhibitor of ROMK1-WNK4 interaction to examine this question. A 3-fold overexpression of $\mathrm{WNK}_{1-444}$ (relative to the amount used in Figure 5A) but without exogenous WNK1 or WNK4 increased ROMK1 currents (Figure 7B). ROMK1 expressed in HEK cells is partially inhibited by endogenous WNKs (13). The cause of the above finding is likely that WNK4 ${ }_{1-444}$ reversed the inhibition by endogenous WNKs. Consistent with this idea, overexpression of $\mathrm{WNK} 4_{1-444}$ reversed the inhibition of ROMK1 caused by exogenous WNK1 $1_{1-491}$ or WNK41-584 (Figure 7B). Overall, our results suggest that interaction with ROMK1 is necessary - although not alone sufficient - for WNK4 regulation of the channel. We further compared the interaction of ROMK1 with wild-type and disease-causing mutants of WNK4. H6-tagged wild-type WNK44-584, E559K, D561A, and Q562E were used to pull down full-length ROMK1. Each mutant interacted with ROMK1 much more strongly than did wild-type WNK4 $4_{1-584}$ (Figure 7C). Thus, PHA2-causing mutations of WNK4 increase its interaction with ROMK1 as well as with ITSN.

Localization of ITSN1 to the distal nephron. ROMK1 channels are present in the distal nephron, including the distal convoluted tubule (DCT), the connecting tubule (CNT), and the cortical collecting duct (CCD), and are important for basal $\mathrm{K}^{+}$secretion in these segments (29). We examined the distribution of ITSN1 in rat kidney by immunofluorescent staining. The staining for ITSN1 by a specific anti-ITSN1 antibody was strong in the distal nephron, including DCT and CCD (Figure 8A, arrowhead and arrow, respectively), but was not apparent in the proximal tubule or glomerulus. No staining was observed without the anti-ITSN1 antibody (Figure $8 \mathrm{~B}$ ). Sodium-calcium exchanger isoform 1 (NCX1) is present in the basolateral membrane of DCT and CNT (30). Aquaporin-2 (AQP2) is present in the apical membrane of CNT and CCD (31). Staining for ITSN was present in NCX1-positive tubules (Figure 8C) and in AQP2-positive tubules (Figure 8D). The patterns of cellular and subcellular staining observed for ITSN1, NCX1, and AQP2 are consistent with the idea that ITSN1 is predominantly distributed intracellularly and near the apical membrane of DCT, CNT, and CCD. WNK1 and WNK4 are also present in the distal nephron (3). These results support the hypothesis that ITSN1 in important for endocytosis of ROMK1 by WNKs. 

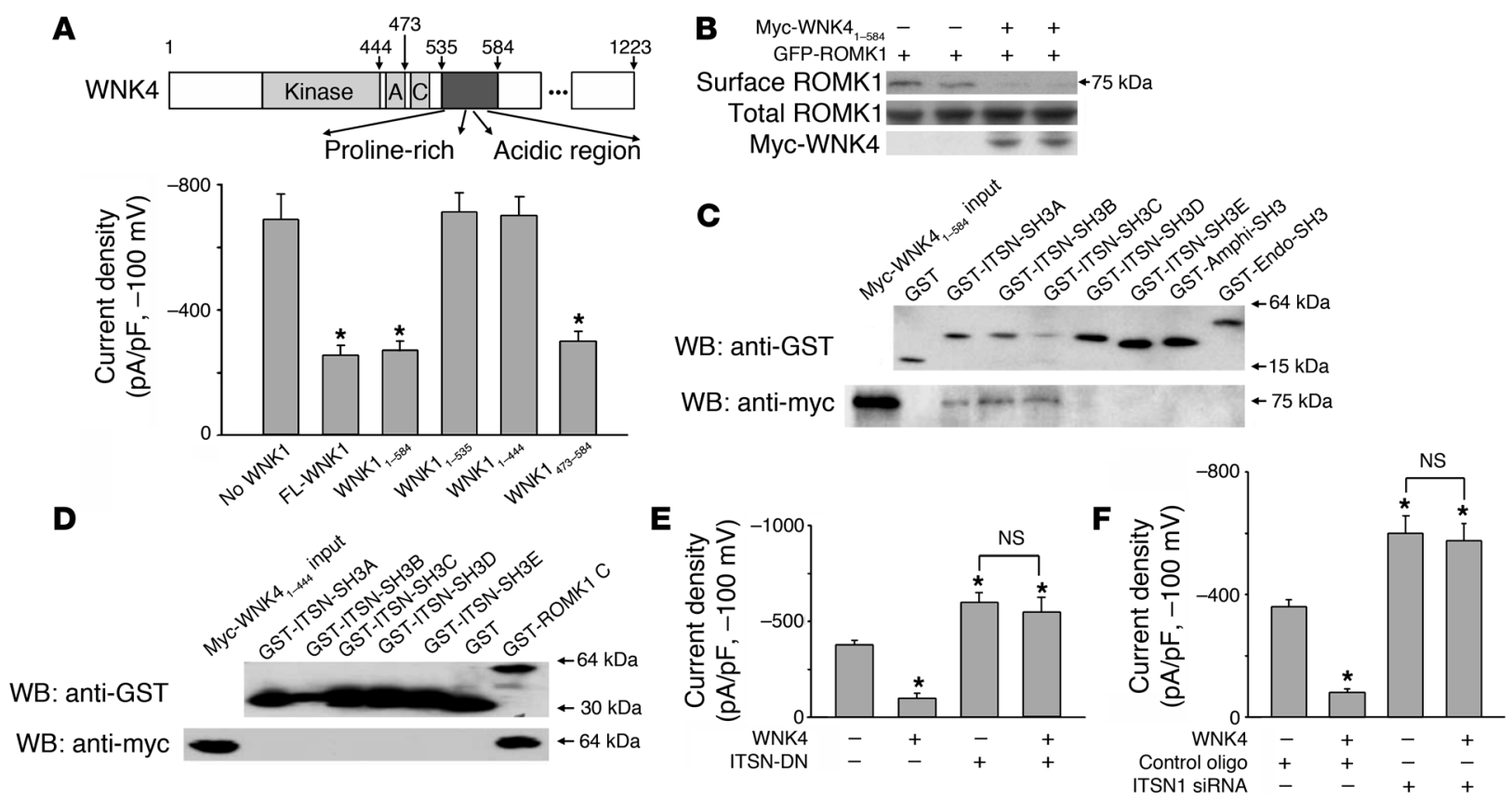

\section{Figure 5}

WNK4 decreases ROMK1 surface abundance via ITSN. (A) Domains of WNK4 involved in regulation of ROMK1 current density. Cells were transfected with ROMK1 plus indicated constructs. A, autoinhibitory domain; C, coiled-coil domain. (B) WNK4 $4_{1-584}$ decreased ROMK1 surface abundance. (C) WNK4 ${ }_{1-584}$ interacted with ITSN SH3A, SH3B, and SH3C. GST fusion proteins were used to pull down myc-tagged WNK4 ${ }_{1-584}$ from lysates of transfected cells. (D) WNK4 $4_{1-444}$ did not interact with ITSN. GST fusion proteins were used to pull down myc-tagged WNK4 ${ }_{1-444}$ from lysates of transfected cells. ROMK1 C, C terminus of ROMK1. (E) A dominant-negative ITSN1 containing SH3A-SH3E increased baseline ROMK1 currents and prevented the decrease caused by WNK4 ${ }_{1-584 .}(\mathbf{F})$ Knockdown of ITSN1 increased baseline ROMK1 currents and prevented the decrease caused by WNK4 $4_{1-584 .}{ }^{*} P<0.05$ versus ROMK1 alone.

ITSN1s and the ITSN1 long form are alternative splice variants containing identical EH, coiled-coil, and SH3 domains. Our antibody raised against the $\mathrm{SH} 3$ domains recognizes both long and short forms. ITSN1s is ubiquitously expressed whereas the long form is predominantly in neuronal tissues (22-24). Thus, ITSN1s is likely the ITSN1 isoform that is important for regulation of ROMK1 by WNKs in the kidney.

\section{Discussion}

PHA2 is characterized by hypertension, hyperkalemia, and hyperchloremic metabolic acidosis. Recently, several studies have reported that long WNK1 and WNK4 inhibit ROMK1 by stimulating clathrin-mediated endocytosis of the channel (12-15). PHA2-causing mutations of WNK4 increase the inhibition of ROMK1 by WNK4 (12), suggesting that the increased endocytosis of ROMK1 may contribute to hyperkalemia in PHA2. How long WNK1 and WNK4 stimulate clathrin-mediated endocytosis of ROMK1 is unknown. Endocytosis via CCVs requires concerted actions of many accessory proteins $(19,20)$. ITSN is a multimodular scaffold protein that recruits endocytic proteins to the clathrin-coated pits $(22,32-34)$. There are 2 ITSN genes, each producing alternatively spliced long and short variants (22-24). Both long and short forms have $\mathrm{EH}$ and SH3 domains. The EH domains of ITSN bind epsin, which targets ITSN to the clathrin coat $(22,32)$. The SH3 domains of ITSN bind synaptojanin and dynamin $(22,32,33)$. Dynamin further recruits amphiphysin, endophilin, and syndapin $(19,20)$. Consistent with the notion that the scaffolding role of ITSN is crucial for endocytosis, flies with mutation of Dap160, the Drosophila homology of ITSN1s, are defective in synaptic vesicle endocytosis $(34,35)$. The levels of dynamin, endophilin, and synaptojanin at nerve terminals are markedly reduced in mutant flies $(34,35)$.

In the present study, we found that long WNK1 and WNK4 specifically interacted with the endocytic scaffold protein ITSN1 by binding to its $\mathrm{SH} 3$ domains. The interaction with ITSN1 was crucial for the endocytosis of ROMK1 stimulated by long WNK1 and WNK4. Mutations of WNK1 in PHA2 are large deletions of intron-1 that result in increased expression of long WNK1 (3), which would likely lead to increased endocytosis of ROMK1 via ITSN1 and contribute to hyperkalemia. This is likely mediated by ITSN1s, which is ubiquitously expressed (22-24). The long form is predominantly in neuronal tissues. Most WNK4 mutations in PHA2 are missense mutations of amino acids localized immediately distal to the first coiled-coil domain (3). We found that missense mutations of WNK4 enhanced the ability of WNK4 to interact with ITSN1 as well as with ROMK1. WNK4 interacts with ROMK1 (12). We found that the interaction with ROMK1, though not in itself sufficient, was essential for WNK4 stimulation of endocytosis of ROMK1. These results suggest that the increased endocytosis of ROMK1 caused by PHA2 mutations of WNK4 may be due to enhanced interactions of WNK4 with ROMK1 and ITSN1. ROMK1 is targeted to coated pits via a C-terminal NPXY motif (16). Interaction with WNK4 may also help localization of ROMK1 to coated pits.

WNK1 and WNK4 regulate a growing list of proteins. Regulation of some of these proteins, including claudins, synaptotagmin 2, and oxidative stress-response kinase 1 (OSR1) and related kinases, 

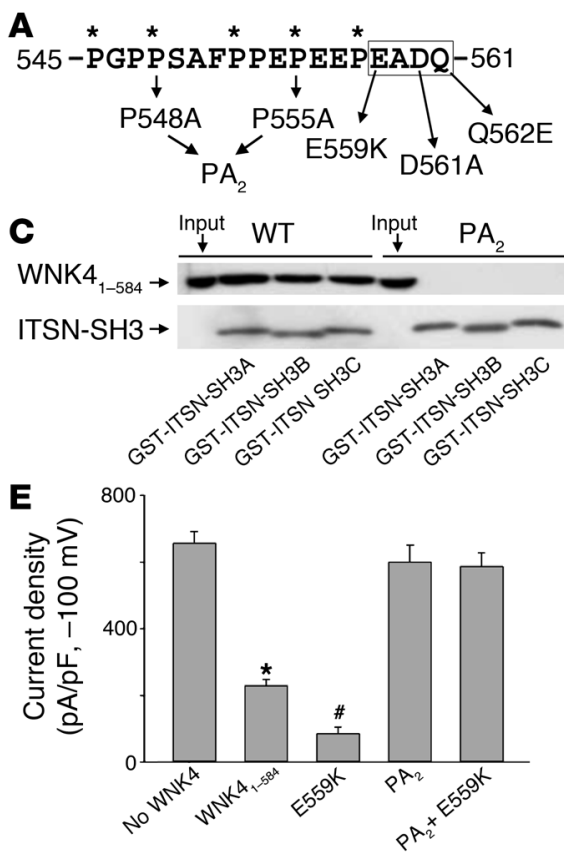

$\mathbf{B}$

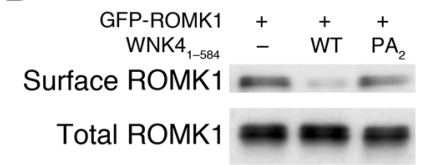

D

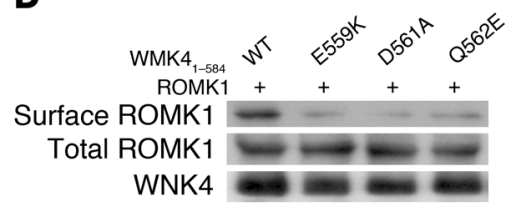

$\mathbf{F}$

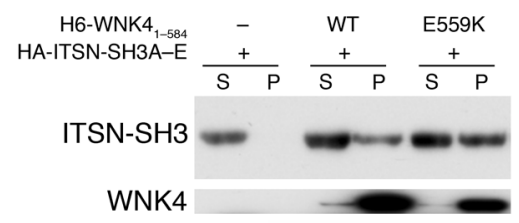

\section{Figure 6}

Representative experiments showing the effects of PHA2 mutation on WNK4 regulation of ROMK1 and interaction with ITSN. (A) Amino acids 545-561 of WNK4 contain 3 PXXP motifs and disease-causing mutations. Double mutations of P548A/P555A disrupted all 3 PXXP motifs (denoted $\mathrm{PA}_{2}$ ). (B) Triple PXXP mutations prevented the decrease of surface ROMK1 caused by WNK41-584. (C) ITSN did not interact with triple PXXP mutations of WNK41-584. GST fusion proteins were used to pull down myc-tagged WT WNK41-584 or WNK4 ${ }_{1-584}$ with the triple PXXP mutation from lysates of transfected cells. (D) Diseasecausing mutations increased the ability of WNK4 to decrease surface ROMK1 levels. (E) Effects of PXXP motifs and disease-causing mutations of WNK4 on ROMK1 currents. Cells were transfected with ROMK1 plus indicated constructs. ${ }^{*} P<0.05$ versus ROMK1 alone. ${ }^{\#} P<0.05$ versus ROMK1 plus WNK4 ${ }_{1-584}$. (F) SH3A-SH3E interacted with WT WNK4 ${ }_{1-584}$ and the E559K mutant. H6-tagged WNK4 ${ }_{1-584}$ protein was used to pull down HA-tagged ITSN SH3A-SH3E from lysates of transfected cells. The amount of ITSN SH3 shown for supernatant $(\mathrm{S})$ and pellet $(\mathrm{P})$ represent $2 \%$ and $50 \%$ of their totals, respectively. To compare the efficacy of ITSN SH3 domain pulldown by WT WNK4 ${ }_{1-584}$ and E559K, the intensity of ITSN SH3 band in pellet was quantified and normalized to that in supernatant (WNK4 ${ }_{1-584}$, 0.32 ; WNK4 ${ }_{1-584}$ plus E559K, 0.83). This is in spite of that the intensity of E559K mutant (used for pulldown) was $65 \%$ that of WT (mean \pm SEM, WNK4 ${ }_{1-584}, 0.38 \pm 0.15$; WNK4 ${ }_{1-584}$ plus E559K, $0.81 \pm 0.21 ; P<0.05 ; n=4$ per group).

require kinase activity of WNKs (9, 10, 36-38). Regulation of $\mathrm{ENaC}$ by long WNK1 is kinase independent $(11,39)$. With respect to ROMK1, Kahle et al. reported that the regulation by WNK4 is independent of its kinase activity (12). However, mutation of the catalytic lysine or the conserved D368 within the kinase domain of WNK1 prevents its regulation of ROMK1, suggesting that the kinase domain plays some roles $(13,14)$. In the present study, we confirmed that the kinase domain of long WNK1 was important for regulation of ROMK1. The regulation of ROMK1 by long WNK1, however, was clearly independent of its catalytic activity. The kinase core of long WNK1, like many protein kinases crystallized to date, adopts a bilobal architecture: an $\mathrm{N}$-terminal lobe composed of mainly $\beta$ strands and a C-terminal lobe of predominantly $\alpha$ helices (27). The interface of the 2 lobes forms the catalytic site. Our results suggest that in long WNK1 an ionic interaction between K233 and D368 in the catalytic site stabilizes the kinase domain fold, allowing the PXXP motifs preceding the kinase domain to interact with ITSN.
Long WNK1 and WNK4 each contain more than 15 PXXP type proline-rich motifs distributed in several clusters along the length of the peptide chain (1-3). Yet we found that only PXXP proline-rich motifs clustered between amino acids 94 and 117 of long WNK1 and between amino acids 545 and 558 of WNK4 are critical for interaction with $\mathrm{SH} 3$ domains of ITSN. Interestingly, the PXXP motifs of WNK4 critical for interaction with ITSN were located right in front of the region of 4 amino acids (EADQ, 559-562) where PHA2-causing mutations are clustered. We found that mutations of the PXXP motifs between amino acids 545 and 558 of WNK4 abolished the enhanced interaction with ITSN and the increased endocytosis of ROMK1 elicited by disease-causing mutations of WNK4. Thus, the ability of these PXXP motifs of WNK4 to interact with ITSN $\mathrm{SH} 3$ domains was influenced by amino acids 559-562, where disease-causing mutations are clustered. Mutations of amino acids 559-562 enhanced WNK4-ITSN interaction.

Amino acids 559-562 of the disease-causing region of WNK4 are conserved in WNK1 (3). To date, there are no reports of missense mutations in this region of WNK1 in patients of PHA2. Notably, the 3 conserved PXXP motifs located in front of the conserved EADQ amino acids 559-562 of WNK4 are not present in long WNK1. Rather, we found that in long WNK1, the PXXP motifs in the $\mathrm{N}$ terminus preceding the kinase domain were critical for interaction with ITSN. The general principle governing context-specific interactions between PXXP motifs and SH3 domains remains elusive.

Regarding context-specific interactions between PXXP motifs and SH3 domains, the SH3 domains of endocytic proteins have different ligand specificities $(22,33)$ and participate at different steps of CCV formation (24). For example, ITSN SH3A, SH3C, and SH3E interact with dynamin stronger than do SH3B and SH3D $(22,26$, 33). ITSN SH3A is required for early events leading to formation of constricted pits, whereas other $\mathrm{SH} 3$ domains are preferentially involved in the late events leading to CCV fission (26). We found that long WNK1 interacted with ITSN SH3C, whereas WNK4 interacted with $\mathrm{SH} 3 \mathrm{~A}, \mathrm{SH} 3 \mathrm{~B}$, and $\mathrm{SH} 3 \mathrm{C}$, raising the possibility that WNKs may be important in coordinating the recruitment of endocytic proteins by ITSN at different stages of CCV formation. The differential interactions with ITSN may also underlie the synergism between long WNK1 and WNK4 stimulating endocytosis of ROMK1 (13).

Long WNK1 is ubiquitous (1). WNK4 also has broad tissue distribution (40). A recent siRNA-based genome-wide screen using virus entry as a measure of clathrin-mediated endocytosis identifies WNK4 as a target (41). Knockdown of long WNK1 also appears to decrease the endocytosis (41). Thus, WNKs may be general players in the endocytic cascades acting upstream of ITSN. 


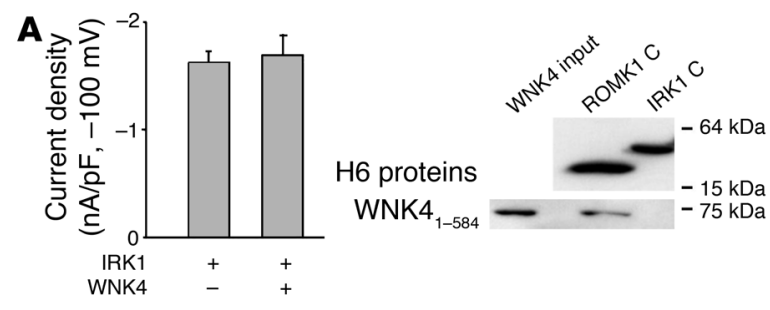

B

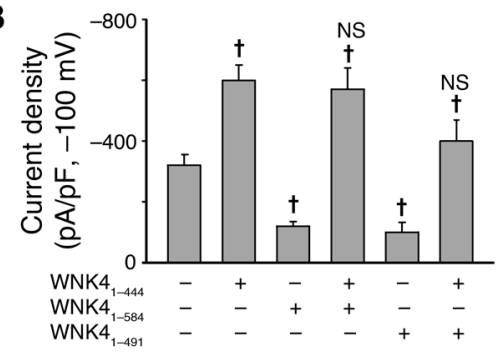

C

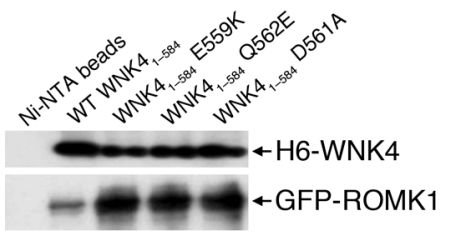

\section{Methods}

Molecular biology. GFP-ROMK1 was described previously (13). Full-length cDNAs encoding rat long WNK1 and WNK4 (gifts of M. Cobb and B. Xu, University of Texas Southwestern Medical Center) were cloned in pCMV5-myc vector $(1,11,13,36,38,39,42)$. WNK1 and WNK4 fragments were amplified by PCR and subcloned into pCMV5-myc. Point mutations were generated by site-directed mutagenesis (QuickChange kit; Stratagene) and confirmed by sequencing. GST- and H6-tagged bacterial fusion proteins containing the $\mathrm{C}$ terminus of ROMK1 and IRK1 have been described previously (43). Plasmids encoding GST fusion proteins containing SH3 domains of ITSN (Xenopus laevis), endophilin (human), and amphiphysin (human; all gifts of P. McPherson, McGill University, Montreal, Quebec, Canada) have also been described previously (26). Sense and antisense oligonucleotides (Dharmacon RNA Technology) for long WNK1 siRNA were 5'-UGUCUAACGAUGGCCGCUU dT dT and 5'-AAGCGGCCAUCGUUAGACA dT dT, respectively (13). Sense and antisense oligonucleotide for ITSN siRNA were 5'-GGACAUAGUUGUACUGAAAUU and 5'-PUUUCAGUACAACUAUGUCCUU, respectively.

Pullown and biotinylation assays. HEK293 cells were cultured and transfected as described previously (13). For pulldown of WNK1 and WNK4 fragments by SH3 domains, cells transfected with myc-tagged WNK plasmids were lysed in an isotonic PBS buffer (1 $\mathrm{ml}$ per 6-well dish) containing phosphatase and protease inhibitors. Cell lysates $(500 \mu \mathrm{g})$ were incubated

\section{Figure 8}

Localization of ITSN1 in rat kidney. (A) Cortical section stained by antiITSN antibody. Fluorescent image was merged with differential interference contrast image. White arrow and arrowhead indicate a CCT and a DCT, respectively. G, glomerulus; P, proximal tubule. Scale bar is shown. (B) Cortical section stained as in panel A, but without the anti-ITSN antibody. (C) Cortical section double-stained by antibodies against ITSN (red) and NCX1 (green). (D) Cortical section doublestained by antibodies against ITSN (red) and AQP2 (green). Scale bars: $50 \mu \mathrm{m}$ (A and $\mathbf{B}) ; 10 \mu \mathrm{m}$ (C and $\mathbf{D})$.

\section{Figure 7}

Role of direct interaction with ROMK1 in the regulation by WNK4. (A) WNK4 ${ }_{1-584}$ interacts with the $\mathrm{C}$ terminus of ROMK1 but not with the $\mathrm{C}$ terminus of IRK1. H6-tagged ROMK1 and IRK1 C termini were used to pull down myc-tagged WNK $1_{1-584}$ from transfected cell lysates. Western blot analysis of proteins was performed using anti-H6 and anti-myc antibody. WNK4 ${ }_{1-584}$ had no effects on IRK1 current density. (B) Effects of various combinations of WNK1 and WNK4 constructs on ROMK1 currents. ${ }^{\dagger} P<0.01$ versus ROMK1 alone. NS, not significantly different versus ROMK1 plus WNK4 ${ }_{1-444}$. Modified statistics adjusted for multiple comparisons were used (see Methods). (C) Interaction between disease-causing WNK4 mutants and full-length ROMK1. Detergentsolubilized GFP-ROMK1 was incubated with WNK4 fusion proteins. ROMK1 was present in all 5 conditions. Without WNK4, nickel beads alone (Ni-NTA beads) did not pull down ROMK1.

with GST fusion proteins of interest ( $1 \mu \mathrm{g}$ in $0.5 \mathrm{ml}$ final volume), and precipitated by glutathione-4B sepharose beads (43). Pulldown of WNKs was examined using an anti-myc antibody (13). Fusion proteins were detected by an anti-GST antibody. For pulldown of full-length ROMK1 by H6-tagged WNK4 fragments, cells transfected with GFP-ROMK1 were lysed in PBS containing 1\% Triton X-100. Pulldown of GFP-ROMK1 was examined using anti-ROMK1 antibody (43). Pulldown of HA-tagged mouse ITSN1 SH3A-SH3E (gift of J. O'Bryan, University of Illinois at Chicago, Chicago, Illinois, USA) by H6-tagged WNK4 fragments was examined using an anti-HA antibody. WNK4 fragments were detected by an anti-H6 antibody. Endogenous long WNK1 and ITSN1s were detected using antiWNK1 antibody (1) (gift of M. Cobb) and anti-ITSN1 antibody (gift of J. O'Bryan), respectively. The anti-ITSN1 antibody was raised against SH3 domains of mouse ITSN1.

For biotinylation of surface ROMK1, cells were washed twice with ice-cold PBS before incubation with $1 \mathrm{ml}$ PBS containing $1 \mathrm{mg} / \mathrm{ml}$ EZ-link-NHS-SSBiotin (Pierce) for 1 hour at $4{ }^{\circ} \mathrm{C}$. After quenching with glycine $(100 \mathrm{mM})$, cells were lysed in PBS containing 1\% Triton X-100. Lysates (500 $\mu \mathrm{g}$ in $1 \mathrm{ml}$ reaction volume) were mixed with $100 \mu \mathrm{l}$ streptavidin beads (Pierce) and incubated for 1 hour. Beads were subsequently washed 3 times with PBS plus $1 \%$ Triton X-100. Biotin-labeled proteins were eluted in sample buffer, separated by SDS-PAGE, and transferred to nitrocellulose membranes for
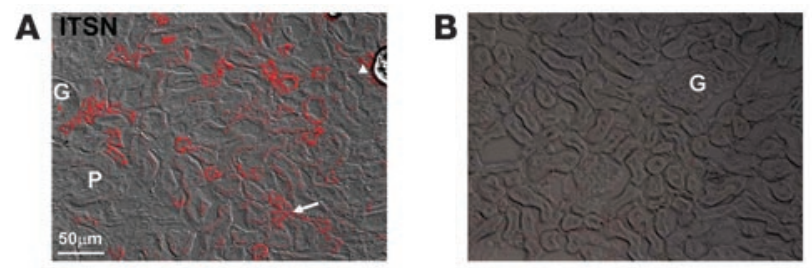

C
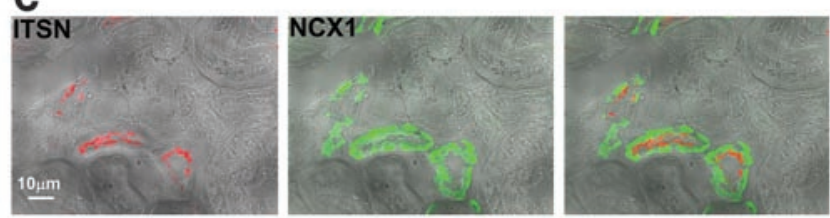

D
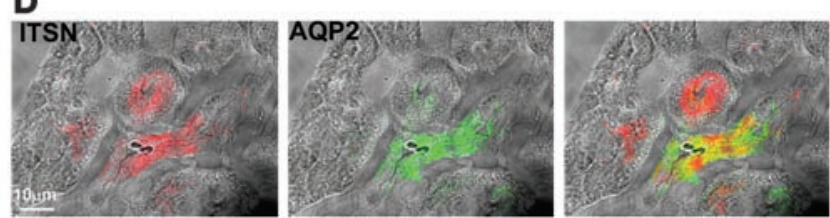
Western blotting. ROMK1 proteins on the membrane were detected with a rabbit polyclonal anti-ROMK1 antibody (43). Each experiment shown was performed at least 3 times with similar results. The intensity of protein bands in Western blot analysis was digitized and quantified using Scion Image analysis software (alpha 4.0.3.2; http://www.scioncorp.com/frames/ fr_download_now.htm; Scion Corporation).

In vitro WNK1 kinase assays. Myc-tagged long WNK1 fragments were immunoprecipitated from lysates of transfected cells using an anti-myc antibody (13) and washed 3 times with a solution containing $1 \mathrm{M} \mathrm{NaCl}$ and $20 \mathrm{mM}$ Tris- $\mathrm{HCl}$ ( $\mathrm{pH} 7.4$ ) and once with a solution containing $10 \mathrm{mM}$ $\mathrm{MgCl}_{2}$ and $10 \mathrm{mM}$ HEPES (pH 8.0). To measure kinase activity $(37,38)$, immunoprecipitates were incubated in $50 \mu \mathrm{l}$ kinase buffer containing $10 \mathrm{mM}$ HEPES (pH 8.0), $10 \mathrm{mM} \mathrm{MgCl} 2,50 \mu \mathrm{M}$ ATP (5 $\mu \mathrm{Ci}$ of $\left.\left[\gamma^{-32} \mathrm{P}\right] \mathrm{ATP}\right)$, $1 \mathrm{mM}$ benzamidine hydrochloride, $1 \mathrm{mM}$ dithiothreitol, and $3 \mu \mathrm{g}$ purified (kinase-dead) GST-OSR1/K44R (gift of M. Cobb and A. Anselmo, University of Texas Southwestern Medical Center; ref. 38) at $30^{\circ} \mathrm{C}$ for 30 minutes.

Patch-clamp recording. HEK293 cells were cotransfected with cDNAs encoding GFP-ROMK1 and full-length or fragments of WNK1 or WNK4 plus additional constructs as indicated (ITSN siRNA in Figure 2E and Figure 5F and ITSN-DN in Figure 2F and Figure 5E). In each experiment, the total amount of DNA for transfection was balanced using an empty vector. About 36-48 hours after transfection, whole-cell currents were recorded using an Axopatch 200B amplifier (Molecular Devices) as previously described (13). Transfected cells were identified using epifluorescent microscopy. The pipette solution contained $140 \mathrm{mM} \mathrm{KCl}$ and $10 \mathrm{mM}$ HEPES ( $\mathrm{pH}$ 7.2); the bath solution contained $140 \mathrm{mM} \mathrm{KCl}, 1 \mathrm{mM} \mathrm{MgCl}_{2}, 1 \mathrm{mM} \mathrm{CaCl}_{2}, 10 \mathrm{mM}$ HEPES ( $\mathrm{pH}$ 7.4). Capacitance and access resistance were monitored and $75 \%$ compensated. The voltage protocol consisted of $0 \mathrm{mV}$ holding potential and $400-\mathrm{ms}$ steps from -100 to $100 \mathrm{mV}$ in $20-\mathrm{mV}$ increments. Current density was calculated by dividing current at $-100 \mathrm{mV}$ (pA; measured at $\left.25^{\circ} \mathrm{C}\right)$ by capacitance $(\mathrm{pF})$. Results were shown as mean $\pm \operatorname{SEM}(n=5-10)$. For cell-attached single-channel recording of ROMK1, the pipette solution contained $140 \mathrm{mM} \mathrm{KCl}, 1 \mathrm{mM} \mathrm{MgCl}, 1 \mathrm{mM} \mathrm{CaCl}_{2}$, and $10 \mathrm{mM}$ HEPES ( $\mathrm{pH}$ 7.4). Single-channel recording and analysis of single-channel conductance and open probability were performed as described previously (44).

Immunofluorescent staining. Sprague-Dawley rats (approximately $250 \mathrm{~g}$, of either sex) were anesthetized and perfused via the heart with $100 \mathrm{ml}$ PBS followed by $50 \mathrm{ml}$ 4\% paraformaldehyde in PBS. Kidneys were harvested, fixed in $4 \%$ paraformaldehyde at $4^{\circ} \mathrm{C}$ for 4 hours, dehydrated by immersion in $30 \%$ sucrose in PBS at $4^{\circ} \mathrm{C}$ overnight, and mounted in OCT (Tissue-Tek; Sakura) for sectioning. Sections $(3-5 \mu \mathrm{m})$ were dried at $37^{\circ} \mathrm{C}$ for 30 minutes, treated with $0.1 \%$ Triton for 10 minutes at room tempera- ture, and quenched in $0.1 \% \mathrm{NaBH}_{4}$ for 30 minutes at room temperature followed by extensive washing in PBS. After blocking with $10 \%$ goat or donkey serum in PBS for 2 hours, sections were incubated overnight at $4{ }^{\circ} \mathrm{C}$ with primary antibodies, which included rabbit polyclonal antibodies against ITSN1 (1:200; gift of J. O'Bryan), mouse monoclonal antibodies against NCX1 (1:400; gift of K. Philipson, University of California at Los Angeles, Los Angeles, California, USA), and goat polyclonal antibodies against AQP2 (1:200; Santa Cruz Biotechnology Inc.). After washing, specimens were incubated with secondary antibodies Alexa Fluor 568conjugated goat anti-rabbit (diluted 1:500; Invitrogen) or fluorescein-conjugated donkey anti-goat (diluted 1:500; Jackson ImmunoResearch Laboratories Inc.) for 30 minutes at room temperature. Fluorescent images were obtained using a Nikon Eclipse TE2000-U fluorescent microscope and overlaid with differential interference contrast images, as we have previously described $(16,18)$. All procedures were performed in compliance with relevant laws and institutional guidelines and were approved by the University of Texas Southwestern Medical Center at Dallas Institutional Animal Care and Use Committee.

Statistics. Statistical comparisons between 2 groups of data were made using 2-tailed unpaired Student's $t$ test. Multiple comparisons were made using 1-way ANOVA followed by 2-tailed Student's $t$ test adjusted for multiple comparisons. $P$ values less than 0.05 and 0.01 were considered significant for single and multiple comparisons, respectively.

\section{Acknowledgments}

We thank Melanie Cobb, Peter McPherson, John O'Bryan, and Kenneth Philipson for plasmids and antibodies; Melanie Cobb, Donald Hilgemann, and Peter Igarashi for discussion and comments; and Beverley Huet for advice on statistical analysis. This work was supported by NIH grants DK-59530 and DK-54368 (to C.-L. Huang).

Received for publication August 16, 2006, and accepted in revised form January 30, 2007.

Address correspondence to: Chou-Long Huang, University of Texas Southwestern Medical Center, Department of Medicine, 5323 Harry Hines Boulevard, Dallas, Texas 75390-8856, USA. Phone: (214) 648-8627; Fax: (214) 648-2071; E-mail: chou-long.huang@ utsouthwestern.edu.

Guocheng He and Hao-Ran Wang contributed equally to this work.
1. Xu, B., et al. 2000. WNK1, a novel mammalian serine/threonine protein kinase lacking the catalytic lysine in subdomain II. J. Biol. Chem. 275:16795-16801.

2. Verissimo, F., and Jordan, P. 2001. WNK kinases, a novel protein kinase subfamily in multi-cellular organisms. Oncogene. 20:5562-5569.

3. Wilson, F.H., et al. 2001. Human hypertension caused by mutations in WNK kinases. Science. 293:1107-1112.

4. Gordon, R.D. 1986. Syndrome of hypertension and hyperkalemia with normal glomerular filtration rate. Hypertension. 8:93-102.

5. Delaloy, C., et al. 2003. Multiple promoters in the WNK1 gene: one controls expression of a kidneyspecific kinase-defective isoform. Mol. Cell. Biol. 23:9208-9221.

6. O'Reilly, M., Marshall, E., Speirs, H.J., and Brown, R.W. 2003. WNK1, a gene within a novel blood pressure control pathway, tissue-specifically generates radically different isoforms with and without a kinase domain. J. Am. Soc. Nephrol. 14:2447-2456.
7. Yang, C.L., Angell, J., Mitchell, R., and Ellison, D.H. 2003. WNK kinases regulate thiazide-sensitive $\mathrm{Na}-\mathrm{Cl}$ cotransport. J. Clin. Invest. 111:1039-1045. doi:10.1172/JCI200317443.

8. Wilson, F.H., et al. 2003. Molecular pathogenesis of inherited hypertension with hyperkalemia: the $\mathrm{Na}-\mathrm{Cl}$ cotransporter is inhibited by wild-type but not mutant WNK4. Proc. Natl. Acad. Sci. U. S. A. 100:680-684

9. Yamauchi, K., et al. 2004. Disease-causing mutant WNK4 increases paracellular chloride permeability and phosphorylates claudins. Proc. Natl. Acad. Sci. U. S. A. 101:4690-4694.

10. Kahle, K.T., et al. 2004. Paracellular Cl- permeability is regulated by WNK4 kinase: insight into normal physiology and hypertension. Proc. Natl. Acad. Sci.U. S. A. 101:14877-14882.

11. Xu, B., et al. 2005. WNK1 activates SGK1 to regulate the epithelial sodium channel. Proc. Natl. Acad. Sci. U. S. A. 102:10315-10320.

12. Kahle, K.T., et al. 2003. WNK4 regulates the balance between renal $\mathrm{NaCl}$ reabsorption and $\mathrm{K}+$ secretion.
Nat. Genet. 35:372-376.

13. Lazrak, A., Liu, Z., and Huang, C.-L. 2006. Antagonistic regulation of ROMK by long and kidney-specific WNK1 isoforms. Proc. Natl. Acad. Sci. U. S. A. 103:1615-1620.

14. Wade, J.B., et al. 2006. WNK1 kinase isoform switch regulates renal potassium excretion. Proc. Natl. Acad. Sci. U. S. A. 103:8558-8563.

15. Cope, G., et al. 2006. WNK1 affects surface expression of the ROMK potassium channel independent of WNK4. J. Am. Soc. Nephrol. 17:1867-1874.

16. Zeng, W.-Z., et al. 2002. Evidence for endocytosis of ROMK potassium channels via clathrin-coated vesicles. Am. J. Physiol. Renal Physiol. 283:F630-F639.

17. Wei, Y., Bloom, P., Lin, D., Gu, R., and Wang, W.H. 2001. Effect of dietary K intake on apical small-conductance $\mathrm{K}$ channel in CCD: role of protein tyrosine kinase. Am. J. Physiol. Renal Physiol. 281:F206-F212.

18. Chu, P.-Y., Quigley, R., Babich, V., and Huang, C.-L. 2003. Dietary potassium restriction stimulates endocytosis of ROMK in rat cortical collecting duct. Am. J. Physiol. Renal Physiol. 285:F1179-F1187. 
19. Marsh, M., and McMahon, H.T. 1999. The structural era of endocytosis. Science. 285:215-220.

20. Higgins, M.K., and McMahon, H.T. 2002. Snapshots of clathrin-mediated endocytosis. Trends Biochem. Sci. 27:257-263.

21. Kay, B.K., Williamson, M.P., and Sudol, M. 2000. The importance of being proline: the interaction of proline-rich motifs in signaling proteins with their cognate domains. FASEB J. 14:231-241.

22. Yamabhai, M., et al. 1998. Intersectin, a novel adaptor protein with two Eps15 homology and five Src homology 3 domains. J. Biol. Chem. 273:31401-31407.

23. Pucharcos, C., Estivill, X., and de la Luna, S. 2000 Intersectin 2, a new multimodular protein involved in clathrin-mediated endocytosis. FEBS Lett. 478:43-51.

24. Pucharcos, C., Casas, C., Nadal, M., Estivill, X., and de la Luna, S. 2001. Human intersectin genes and their spliced variants are differentially expressed. Biochim. Biophys. Acta. 1521:1-11.

25. Sengar, A.S., Wang, W., Bishay, J., Cohen, S., and Egan, S.E. 1999. The EH and SH3 domain Ese proteins regulate endocytosis by linking to dynamin and Eps15. EMBO J. 18:1159-1171.

26. Simpson, F., et al. 1999. SH3-domain-containing proteins function at distinct steps in clathrin-coated vesicle formation. Nat. Cell Biol. 1:119-124.

27. Min, X., Lee, B.H., Cobb, M.H., and Goldsmith, E.J. 2004. Crystal structure of the kinase domain of WNK1, a kinase that causes a hereditary form of hypertension. Structure. 12:1303-1311.
28. Kubo, Y., Baldwin, T.J., Jan, Y. N., and Jan, L.Y. 1993. Primary structure and functional expression of a mouse inward rectifier potassium channel. Nature. 362:127-133

29. Giebisch, G., and Wang, W. 2000. Renal tubule potassium channels: function, regulation and structure. Acta Physiol. Scand. 170:153-173.

30. Loffing, J., and Kaissling, B. 2003. Sodium and calcium transport pathways along the mammalian distal nephron: from rabbit to human. Am. J. Physiol. Renal Physiol. 284:F628-F643.

31. Nielsen, S., et al. 2002. Aquaporins in the kidney: from molecules to medicine. Physiol. Rev. 82:205-244.

32. Okamoto, M., Schoch, S., and Sudhof, T.C. 1999. $\mathrm{EHSH} 1 /$ intersectin, a protein that contains $\mathrm{EH}$ and $\mathrm{SH} 3$ domains and binds to dynamin and SNAP-25. A protein connection between exocytosis and endocytosis? J. Biol. Chem. 274:18446-18454.

33. Roos, J., and Kelly, R.B. 1998. Dap160, a neural-specific Eps15 homology and multiple SH3 domaincontaining protein that interacts with Drosophila dynamin. J. Biol. Chem. 273:19108-19119.

34. Koh, T.W., Verstreken, P., and Bellen, H.J. 2004. Dap160/intersectin acts as a stabilizing scaffold required for synaptic development and vesicle endocytosis. Neuron. 43:193-205.

35. Marie, B., et al. 2004. Dap160/intersectin scaffolds the periactive zone to achieve high-fidelity endocytosis and normal synaptic growth. Neuron. 43:207-219.

36. Lee, B.H., et al. 2004. WNK1 phosphorylates synaptotagmin 2 and modulates its membrane binding.
Mol. Cell. 15:741-751.

37. Vitari, A.C., Deak, M., Morrice, N.A., and Alessi, D.R. 2005. The WNK1 and WNK4 protein kinases that are mutated in Gordon's hypertension syndrome phosphorylate and activate SPAK and OSR1 protein kinases. Biochem. J. 391:17-24.

38. Anselmo, A.N., et al. 2006. WNK1 and OSR1 regulate the $\mathrm{Na}^{+}, \mathrm{K}^{+}, 2 \mathrm{Cl}$ - cotransporter in HeLa cells. Proc. Natl. Acad. Sci. U. S. A. 103:10883-10888.

39. Xu, B., Stippec, S., Lazrak, A., Huang, C.-L., and Cobb, M.H. 2005. WNK1 activates SGK1 by a PI-3 kinase-dependent and non-catalytic mechanism. J. Biol. Chem. 280:34218-34223.

40. Kahle, K.T., et al. 2004. Paracellular Cl- permeability is regulated by WNK4 kinase: insight into normal physiology and hypertension. Proc. Natl. Acad. Sci.U.S. A. 101:14877-14882.

41. Pelkmans, L., et al. 2005. Genome-wide analysis of human kinases in clathrin- and caveolae/raft-mediated endocytosis. Nature. 436:78-86.

42. Xu, B., et al. 2002. Regulation of WNK1 by an autoinhibitory domain and autophosphorylation. J. Biol. Chem. 277:48456-48462.

43. Huang, C.L., Feng, S., and Hilgemann, D.W. 1998. Direct activation of inward rectifier potassium channels and its stabilization by G $\beta \gamma$. Nature. 391:803-806

44. Zeng, W.-Z., Li, X., Hilgemann, D.W., and Huang, C.-L. 2003. Protein kinase C inhibits ROMK1 channel activity via a phosphatidylinositol-4,5bisphosphate-dependent mechanism. J. Biol. Chem. 278:16852-16856. 